九州大学学術情報リポジトリ

Kyushu University Institutional Repository

\title{
DISCRETE DYNAMIC PROGRAMMING WITH RECURSIVE ADDITIVE SYSTEM
}

Iwamoto, Seiichi

Department of Mathematics, Kyushu University

https://doi.org/10.5109/13082

出版情報：統計数理研究. 16 (1/2)，pp.49-66，1974-03. Research Association of Statistical Sciences

バージョン :

権利関係 : 


\title{
DISCRETE DYNAMIC PROGRAMMING WITH RECURSIVE ADDITIVE SYSTEM
}

\author{
By
}

\author{
Seiichi Iwamoto*
}

(Received July 15, 1973)

\section{Introduction.}

In the paper [5], N. Furukawa and S. Iwamoto have defined Markovian decision processes with a new broad class of reward systems, that is, recursive reward functions, and have studied the existence and properties of optimal policies. Under some conditions on the reward functions, they have proved that there exists a $(p, \varepsilon)$-optimal stationary policy and that in the case of a finite action space there exists an optimal stationary policy. These are some generalizations of results by D. Blackwell [3].

In this paper the author defines a dynamic programming problem with a recursive additive system which is referred to one type of Markovian decision processes with recursive reward functions defined by the previous authors [5]. This paper gives an algorithm for finding optimal stationary policies in the dynamic programming with the recursive additive system in the case of finite state and action spaces. Furthermore, we give several interesting examples with numerical computations to obtain optimal policies.

The motivation to consider the dynamic programming problem with the recursive additive system is the following: If we restrict the "reward" in narrow sense, for instance, the money in economic systems or the loss in statistical decision problems, it will be appropriate for us to accept the total sum of stage-wise rewards as a performance index. That is so-called additive reward system. But many practical problems in the field of engineerings enable us to interpret the "reward" in wider sense. In those problems we often encounter much complicated reward systems that are more than so-called additive. We have an interesting class of such complicated reward systems in which we can find a common feature named "recursive additive". By talking about various reward systems belonging to this class at the same time, we can make clear, as a dynamic programming problem, an important common property within the class, Our proofs are partially owing to Blackwell [2].

\section{Notations and optimization problem.}

A dynamic programming problem with a recursive additive system is, in general, defined by six-tuple $\{S, A, p, r, \beta, t\}$, where $S$ is a set of states labeled by the integers

\footnotetext{
* Department of Mathematics, Kyushu University, Fukuoka.
} 
$s=1,2, \cdots, N$, that is, $S=\{1,2, \cdots, N\}, A$ is a set of actions labeled by the integers $a=1,2, \cdots, K$, that is, $A=\{1,2, \cdots, K\}, p$ is a transition law $p_{i j}^{k}$, that is,

$$
\sum_{j=1}^{N} p_{i j}^{k}=1, \quad p_{i j}^{k} \geqq 0 \quad \text { for } \quad i, j \in S, k \in A,
$$

$r=\left(r_{i j}^{k} ; i, j \in S, k \in A\right)$ is a set of stage-wise rewards, $\beta=\left(\beta_{i j}^{k} ; i, j \in S, k \in A\right)$ is a generalized accumulator whose value $\beta_{i j}^{k}$ is a discount factor depending on transition $(i, k, j)$, and $t$ is a translator from $R^{1}$ to $R^{1}$.

Throughout this paper we call the dynamic programming problem with recursive additive system "recursive additive dynamic programming" or simply " recursive additive DP". We sometimes use the convenient notations $\beta(i, k, j), r(i, k, j)$ and $p(i, k, j)$ in stead of $\beta_{i j}^{k}, r_{i j}^{k}$ and $p_{i j}^{k}$ respectively.

When the system starts from initial state $s_{1} \in S$ at 1 -st stage and the decision maker takes an action $a_{1} \in A$ on this state $s_{1}$, the system moves to next state $s_{2} \in S$ with probability $p\left(s_{1}, a_{1}, s_{2}\right)$ at 2-nd stage and the system yields a stage-wise reward $r\left(s_{1}, a_{1}, s_{2}\right)$ and a discount factor $\beta\left(s_{1}, a_{1}, s_{2}\right)$.

However, at the end of 1 -st stage the decision maker indeed gets the translated reward $t\left(r\left(s_{1}, a_{1}, s_{2}\right)\right)$. The system is then repeated from the new state $s_{2} \in S$ at 2-nd stage. If he chooses an action $a_{2} \in A$ on state $s_{2}$, it moves to state $s_{3}$ with probability $p\left(s_{2}, a_{2}, s_{3}\right)$ at 3 -rd stage. Then the system also yields a stage-wise reward $r\left(s_{2}, a_{2}, s_{3}\right)$ and a discount factor $\beta\left(s_{2}, a_{2}, s_{3}\right)$ at the end of 2-nd stage, and he really receives the discounted reward $\beta\left(s_{1}, a_{1}, s_{2}\right) t\left(r\left(s_{2}, a_{2}, s_{3}\right)\right)$ of the translated one $t\left(r\left(s_{2}, a_{2}, s_{3}\right)\right)$ multiplied by a discount factor $\beta\left(s_{1}, a_{1}, s_{2}\right)$ which was swept at the end of 1-st stage. Similarly at the end of 3-rd stage he gets a reward $\beta\left(s_{1}, a_{1}, s_{2}\right) \beta\left(s_{2}, a_{2}, s_{3}\right) t\left(r\left(s_{3}, a_{3}, s_{4}\right)\right)$ which is discounted one of $t\left(r\left(s_{3}, a_{3}, s_{4}\right)\right)$ multiplied by $\beta\left(s_{1}, a_{1}, s_{2}\right) \beta\left(s_{2}, a_{2}, s_{3}\right)$. In general when he undergoes the history $\left(s_{1}, a_{1}, s_{2}, a_{2}, \cdots, s_{n}, a_{n}, s_{n+1}\right)$ of the system up to $n$-th stage, he comes to receive a reward $\beta\left(s_{1}, a_{1}, s_{2}\right) \beta\left(s_{2}, a_{2}, s_{3}\right) \cdots \beta\left(s_{n-1}, a_{n-1}, s_{n}\right) t\left(r\left(s_{n}, a_{n}, s_{n+1}\right)\right)$ at the end of $n$-th stage. Furthermore, the process goes on $(n+1)$-st stage, $(n+2)$-nd stage and so on.

Since we consider a sequential nonterminating decision process, the decision maker continues to take actions infinitely. Consequently if he undergoes the history $h=\left(s_{1}, a_{1}, s_{2}, a_{2}, \cdots\right)$, he comes to receive the total reward

$$
\begin{aligned}
V(h)= & t\left(r\left(s_{1}, a_{1}, s_{2}\right)\right)+\beta\left(s_{1}, a_{1}, s_{2}\right) t\left(r\left(s_{2}, a_{2}, s_{3}\right)\right) \\
& +\beta\left(s_{1}, a_{1}, s_{2}\right) \beta\left(s_{2}, a_{2}, s_{3}\right) t\left(r\left(s_{3}, a_{3}, s_{4}\right)\right) \\
& +\cdots+\beta\left(s_{1}, a_{1}, s_{3}\right) \beta\left(s_{2}, a_{2}, s_{3}\right) \\
& \cdots \beta\left(s_{n-1}, a_{n-1}, s_{n}\right) t\left(r\left(s_{n}, a_{n}, s_{n+1}\right)\right)+\cdots .
\end{aligned}
$$

This reward system is illustrated as follows: 


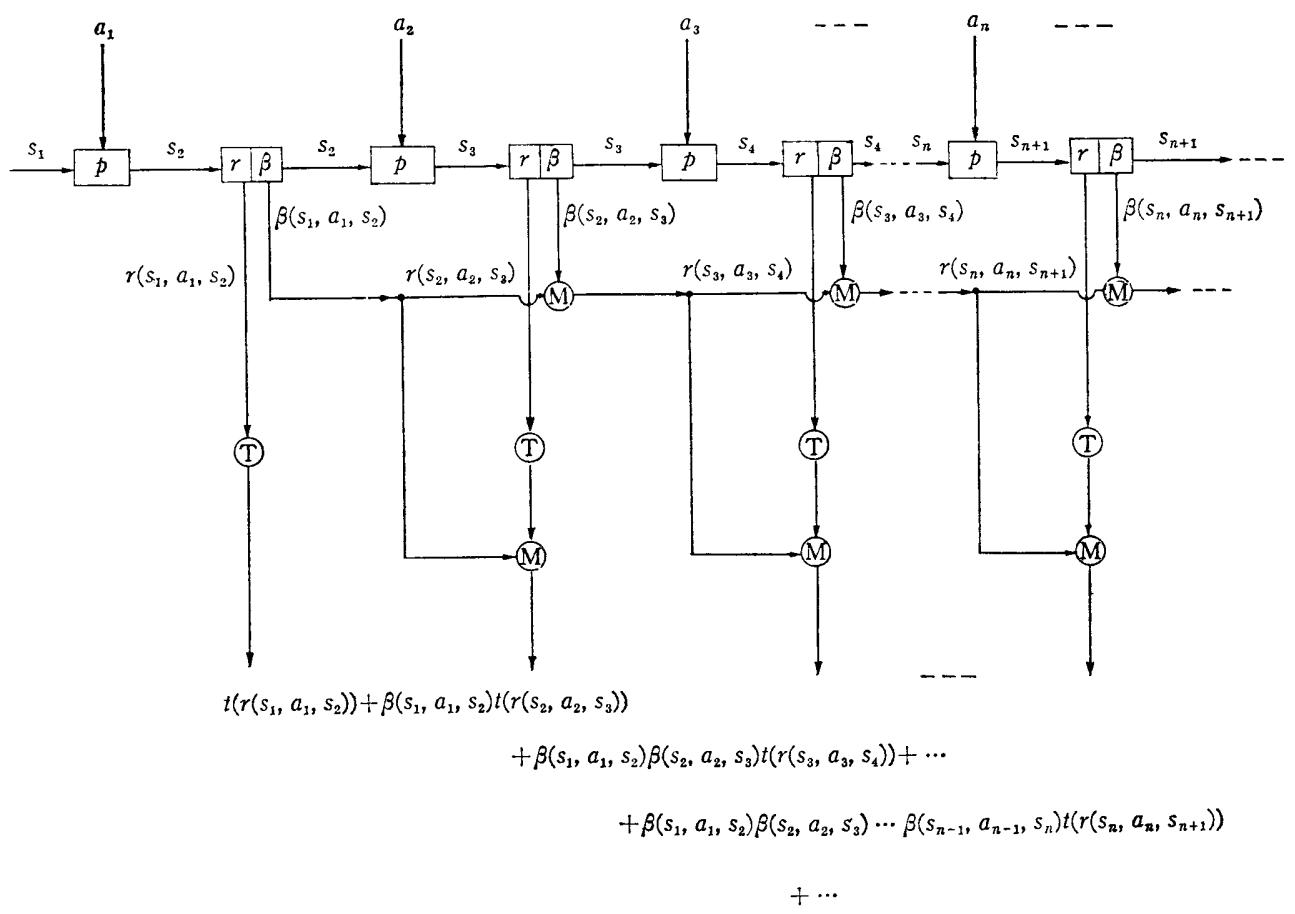

Fig. 1. Reward system of recursive additive DP.

It should be noted that (D), and (ii) mean

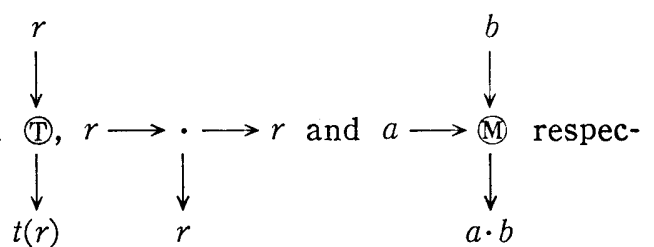

tively in Fig. 1. The decision maker wishes to maximize his total expected reward over the infinite future.

We assume that he has a complete information on his history consisted of states and actions up to date and that he knows not only the stage-wise reward $r=\left(r_{i j}^{k}\right)$, its translator $t=t(\cdot)$ and the generalized accumulator $\beta=\left(\beta_{i j}^{k}\right)$ but also the recursive additivity of reward system in Fig. 1.

Let $F$ denote the (finite) set of all functions from $S$ to $A$. By a policy, we mean a sequence $\left\{f_{n}, n=1,2, \cdots\right\}$ of functions $f_{n} \in F$. We usually write a policy $\pi=$ $\left\{f_{1}, f_{2}, \cdots\right\}$. Using policy $\pi$ means that, if we find the system in state $s$ on the $n$-th day, the action chosen that day is $f_{n}(s) \in A$. A policy $\left\{g, f_{1}, f_{2}, \cdots\right\}$ is denoted by $\{g, \pi\}$. By $\left\{g^{n}, \pi\right\}$, we mean the policy $\left\{g, g, \cdots, g, f_{1}, f_{2}, \cdots\right\}$. For $\pi=\left\{f_{1}, f_{2}, \cdots\right\}$, we define ${ }^{n} \pi$ for each $n$ by ${ }^{n} \pi=\left\{f_{n+1}, f_{n+2}, \cdots\right\}$. In particular ${ }^{0} \pi=\pi$. A policy $\{f, f, \cdots\}$, denoted by $f^{(\infty)}$, is called a stationary policy.

We associate with each $f \in F$ the $N \times 1$ column vector $\bar{r}(f)$ whose $i$-th element $\bar{r}(f)(i)$ is

$$
\tilde{r}(f)(i)=\sum_{j=1}^{N} p_{i j}^{f(i)} t\left(r_{i j}^{f(i)}\right) \quad i=1,2, \cdots, N
$$


and (ii) the $N \times N$ Markov matrix $\vec{P}(f)$ whose $(i, j)$ element $\bar{P}(f)(i, j)$ is

$$
\bar{P}(f)(i, j)=p_{i j}^{f(i)} \times \beta_{i j}^{f(i)} \quad i, j=1,2, \cdots, N .
$$

If the decision maker uses policy $\pi=\left\{f_{1}, f_{2}, \cdots\right\}$ and the system is initially in state $i \in S$, his total expected return from $\pi$ is the column vector

$$
V(\pi)=\sum_{n=0}^{\infty} \bar{P}_{n}(\pi) \bar{r}\left(f_{n+1}\right),
$$

where $\bar{P}_{0}(\pi)=I$, the $N \times N$ identity matrix, and for $n \geqq 1$

$$
\bar{P}_{n}(\pi)=\bar{P}\left(f_{1}\right) \bar{P}\left(f_{2}\right) \cdots \bar{P}\left(f_{n}\right) .
$$

That is, $i$-th element of $V(\pi)$ is

$$
\begin{aligned}
& V(\pi)(i)=\bar{r}\left(f_{1}\right)(i)+\sum_{j=1}^{N} p_{i j}^{f_{1}(i)} \beta_{i j}^{f_{1}^{(i)}} \bar{r}\left(f_{2}\right)(j)+\sum_{j, k=1}^{N} p_{i j}^{f_{1}(i)} p_{j k}^{f^{2}(j)} \beta_{i \bar{j}}^{f_{1}^{(i)}} \beta_{j \bar{k}}^{f_{2}(j)} \bar{r}\left(f_{3}\right)(k) \\
& +\cdots+\sum_{j, k, \cdots, h, l=1}^{N} p_{i j}^{f_{1}^{1}(i)} p_{j k}^{f_{2}(j)} \cdots p_{h l}^{f_{n-1}(h)} \beta_{i j}^{f_{1}^{(i)}} \beta_{j k}^{f_{2}(j)} \cdots \beta_{h l}^{f_{n-1}(h)} \bar{r}\left(f_{n}\right)(l)+\cdots .
\end{aligned}
$$

We associate with each $f \in F$ the operator $L(f)$ which maps the $N \times 1$ column vector $u$ into $L(f) u=\vec{r}(f)+\bar{P}(f) u$. For any two column vectors $u$, $v$ we write $u \geqq v$ if every coordinate of $u$ is at least as large as the corresponding coordinate of $v$, and $u>v$ if $u \geqq v$ and $u \neq v$. For $N \times 1$ column vector $u=\left(u_{1}, \cdots, u_{N}\right)^{\prime}$ and $N \times N$ matrix $A=\left(a_{i j}\right)$, we use the norms $\|u\|$, $\|A\|$ defined by $\|u\|=\max _{1 \leq i \leq y}\left|u_{i}\right|,\|A\|=$ $\max _{1 \leqq i \leqq N} \sum_{j=1}^{N}\left|a_{i j}\right|$ respectively.

A policy $\pi^{*}$ is called optimal if $V\left(\pi^{*}\right) \geqq V(\pi)$ for all $\pi$.

\section{Optimal policies.}

We now set

Assumption (I). It follows that $0 \leqq \beta_{i j}^{k}<1$ for any $(i, k, j) \in S \times A \times S$. $i \in S$

We remark that if Assumption (I) is satisfied, we have for any policy $\pi$ and any

$$
\frac{r_{*}}{1-K_{*}} \leqq V(\pi)(i) \leqq \frac{r^{*}}{1-K^{*}},
$$

where $r_{*}=\min _{i, k, j} t\left(r_{i j}^{k}\right), r^{*}=\max _{i, k, j} t\left(r_{i j}^{k}\right), K_{*}=\min _{i, k, j} \beta_{i j}^{k}$ and $K^{*}=\max _{i, k, j} \beta_{i j}^{k}$.

Hence Assumption (I) assures the finiteness of the vector $V(\pi)$ for any policy. Furthermore note that Assumption (I) implies the hypotheses in the following Lemmas 3.2 and 3.3 .

Under Assumption (I) Furukawa and Iwamoto (Theorem 6.6 (ii) in [5]) have proved that there exists an optimal policy which is stationary in the finite stateand-action space dynamic programming problem with recursive additive functions.

In this section we shall give an algorithm for finding optimal policies which is stationary.

LEMMA 3.1. (i) For any $f \in F$ and any policy $\pi$ we have 


$$
V(f, \pi)=L(f) V(\pi) .
$$

(ii) For each $f_{n} \in F, n \geqq 1$ and any policy $\pi$ we have

$$
V\left(f_{1}, f_{2}, \cdots, f_{n}, \pi\right)=L\left(f_{1}\right) L\left(f_{2}\right) \cdots L\left(f_{n}\right) V(\pi) .
$$

Proof. The proof of this lemma is easy.

LEMMA 3.2. (i) If $\beta_{i j}^{k} \geqq 0$ for each $(i, k, j) \in S \times A \times S$, then $L(f)$ is monotone, that is, $u \geqq v$ implies $L(f) u \geqq L(f) v$ for each $f \in F$.

(ii) If $\max _{(i, k, j) \equiv S A S}\left|\beta_{i j}^{k}\right|<1$, then for each $f \in F$ the operator $L(f)$ is a contraction

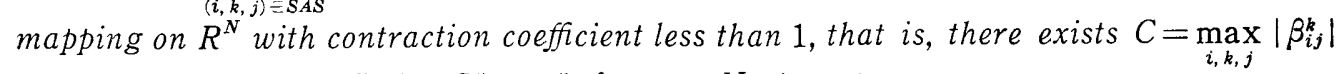
such that $\|L(f) u-L(f) v\| \leqq C\|u-v\|$ for any $N \times 1$ vectors $u, v$.

Proof. The first result follows directly from the equality

$$
L(f) u-L(f) v=\bar{P}(f)(u-v) .
$$

Then, the second follows by the inequalities

and

$$
\begin{aligned}
& \|L(f) u-L(f) v\| \leqq\|\bar{P}(f)\| \times\|u-v\|, \\
& \|\bar{P}(f)\| \leqq \max _{(i, k, j) \in S A S}\left|\beta_{i j}^{k}\right|\|P(f)\|
\end{aligned}
$$

$$
\|P(f)\|=1,
$$

where $\{P(f)(i, j)\}=\left\{P_{i j}^{f(i)}\right\}$ is the Markov matrix for each $f \in F$.

LEMMA 3.3. Let $\max _{i, k, j}\left|\beta_{i j}^{k}\right|<1$ be satisfied. Then it follows that $\lim _{n \rightarrow \infty} L\left(f_{1}\right) L\left(f_{2}\right)$ $\ldots L\left(f_{n}\right) w=V(\pi)$ for any policy $\pi=\left\{f_{1}, f_{2}, \cdots, f_{n}, \cdots\right\}$ and any $w \in R^{N}$.

PROOF. The proof is straightforward and we omit it.

Throughout the reminder of this section, we shall discuss analogously according to Blackwell [2].

THEOREM 3.1. Let Assumption (I) be satisfied. If $V\left(\pi^{*}\right) \geqq V\left(f, \pi^{*}\right)$ for any $f \in F$, then $\pi^{*}$ is optimal.

Proof. By Lemma 3.1 (i), our hypothesis is that

$$
V\left(\pi^{*}\right) \geqq L(f) V\left(\pi^{*}\right) \quad \text { for any } f \in F .
$$

By Lemma 3.2 (i), we have for any policy $\pi=\left\{f_{1}, f_{2}, \cdots, f_{n}, \cdots\right\}$

$$
V\left(\pi^{*}\right) \geqq L\left(f_{1}\right) L\left(f_{2}\right) \cdots L\left(f_{n}\right) V\left(\pi^{*}\right) \quad n \geqq 1 .
$$

Consequently Lemma 3.3, with letting $n \rightarrow \infty$, implies that

$$
V\left(\pi^{*}\right) \geqq V(\pi) .
$$

Since $\pi$ is arbitrary policy, the proof is complete.

THEOREM 3.2. Let Assumption $(I)$ be satisfied. If $V(f, \pi)>V(\pi)$, then $V\left(f^{(\infty)}\right)$ $>V(\pi)$.

Proof. By Lemma 3.1 (i), our hypothesis is that

$$
L(f) V(\pi)>V(\pi) \text {. }
$$

Since Lemma 3.2 (i) holds, that is, operator $L(f)$ is monotone, applying the monotone operator $L^{n-1}(f)$ yields 
so that

$$
L^{n}(f) V(\pi) \geqq L^{n-1}(f) V(\pi),
$$

$$
V\left(f^{n}, \pi\right) \geqq V(f, \pi)>V(\pi) \quad \text { for all } n \geqq 1 \text {. }
$$

Then letting $n \rightarrow \infty$, we have by virtue of Lemma 3.3

$$
V\left(f^{(\infty)}\right)>V(\pi) \text {. }
$$

Now we have our main results which are analogues of the Howard's policy improvement algorithm for the case where $\beta$ depends on $(i, k, j) \in S \times A \times S$.

THEOREM 3.3. Let Assumption $(I)$ be satisfied. Take any $f \in F$. For each $i \in S$, denote $G(i, f)$ the set of all $k \in A$ for which

$$
\bar{r}_{i}^{k}+\sum_{j=1}^{N} p_{i j}^{k} \beta_{i j}^{k} V\left(f^{(\infty)}\right)(j)>V\left(f^{(\infty)}\right)(i),
$$

where $\bar{r}_{i}^{k}=\sum_{j=1}^{N} p_{i j}^{k} t\left(r_{i j}^{k}\right)$. If $G(i, f)$ is empty for all $i \in S$, then $f^{(\infty)}$ is optimal. For any $g \in F$ such that

(a) $g(i) \in G(i, f)$ for some $i$ and

(b) $g(i)=f(i)$ whenever $g(i) \notin G(i, f)$,

we have $V\left(g^{(\infty)}\right)>V\left(f^{(\infty)}\right)$.

Proof. It follows that

$$
V\left(g, f^{(\infty)}\right)(i)=\bar{r}(g)(i)+\bar{P}(g) V\left(f^{(\infty)}\right)(i) .
$$

Furthermore, $V\left(g, f^{(\infty)}\right)(i)>V\left(f^{(\infty)}\right)(i)$ if and only if $g(i) \in G(i, f)$, and $V\left(g, f^{(\infty)}\right)(i)=$ $V\left(f^{(\infty)}\right)(i)$ if $g(i)=f(i)$. Thus if $G(i, f)$ is empty for all $i \in S, V\left(f^{(\infty)}\right) \geqq V\left(g, f^{(\infty)}\right)$ for all $g$ so that, from Theorem 3.1, $f^{(\infty)}$ is optimal. On the other hand, for any $g$ satisfying (a) and (b), we have

$$
V\left(g, f^{(\infty)}\right)>V\left(f^{(\infty)}\right),
$$

so that, from Theorem 3.2, $V\left(g^{(\infty)}\right)>V\left(f^{(\infty)}\right)$.

COROLlaRY 1. Under Assumption (I), there is an optimal policy which is stationary.

PROOF. According to Theorem 3.3, if we take any stationary policy $f^{(\infty)}$, either it is optimal, that is, $G(i, f)$ is empty for all $i \in S$, or it has a stationary improvement $g^{(\infty)}$, that is, $G(i, f)$ is nonempty for some $i \in S$. Since $F$ is finite, there is one which has no stationary improvement with finite interations, so that it must be optimal.

COROllary 2. Let Assumption (I) be satisfied. Take any $f_{1} \in F$. Then solve the linear equation

$$
v_{i}=\bar{r}\left(f_{1}\right)(i)+\sum_{j=1}^{N} \bar{P}\left(f_{1}\right)(i, j) v_{j} \quad j=1,2, \cdots, N
$$

for $v_{i}(i \in S)$. Using these values $v_{i}(i \in S)$, find the element of $G\left(i, f_{1}\right)$ for each $i \in S$. If $G\left(i, f_{1}\right)$ is empty for all $i \in S, f_{1}^{(\infty)}$ is optimal, and $V\left(f_{1}^{(\infty)}\right)(i)=v_{i}$ for all $i \in S$. If at least $f_{2}(i) \in G\left(i, f_{1}\right)$ for some $i \in S$, we obtain an improved policy $f_{2}^{(\infty)}$ such that $f_{2}(i) \in G\left(i, f_{1}\right)$ for some $i$ and $f_{2}(i)=f_{1}(i)$ for $G\left(i, f_{1}\right)$ empty. We then return to solving the linear equation for $f_{2}$. And again we find the element of $G\left(i, f_{2}\right)$ for each $i \in S$. If we repeat this procedure, then for some $n$ we have

$$
f_{n}(i)=f_{n+1}(i) \quad \text { for all } i \in S
$$


and then $f_{n}^{(\infty)}$ is an optimal policy which is stationary.

Proof. The proof follows directly from Theorem 3.3 and Corollary 1. Note that, as an example, we can take an initial $f_{1} \in F$ such that $\bar{r}\left(f_{1}\right)(i)=\max _{1 \leqq k \leqq N} \sum_{j=1}^{N} p_{i j}^{k} t\left(r_{i j}^{k}\right)$.

\section{Application to some additive systems.}

We now illustrate some interesting examples with numerical computation which follow from the discussion in Section 3.

EXAMPLE 1. (General Additive System)

We say that a dynamic programming problem $\{S, A, p, r, \beta\}$ has a general additive system if $t(r)=r$ for any $r \in R^{1}$ in the recursive additive $\operatorname{DP}\{S, A, p, r, \beta, t\}$ discussed at Section 2. We call this dynamic programming "general additive DP". In this case we have an optimization problem where our aim is to maximize the expected reward of the function

$$
V(h)=r_{1}+\beta_{1} r_{1}+\beta_{1} \beta_{2} r_{3}+\cdots+\beta_{1} \beta_{2} \cdots \beta_{n-1} r_{n}+\cdots
$$

over the infinite future and to find the optimal policy among the all ones, where $h=\left(s_{1}, a_{1}, s_{2}, a_{2}, \cdots\right), \quad r_{k}=r_{s_{k} s_{k+1}}^{a_{k}}=r\left(s_{k}, a_{k}, s_{k+1}\right), \quad \beta_{k}=\beta_{s_{k} s_{k+1}}^{a_{k}}=\beta\left(s_{k}, a_{k}, s_{k+1}\right), s_{k} \in S$ and $a_{k} \in A$ for $k \geqq 1$.

In many financial decision problems, the discount factor $\beta_{i j}^{k}$ may depend on selected action $k \in A$. Then we have $K$ different discount factors $\beta_{1}, \beta_{2}, \cdots, \beta_{K}$. These problems can be formulated into the general additive DP.

In particular, if $0 \leqq \beta_{i j}^{k} \equiv \beta<1$, then we have a dynamic programming problem $\{S, A, p, r, \beta\}$ whose objective function is

$$
V(h)=r_{1}+\beta r_{2}+\beta^{2} r_{3}+\cdots+\beta^{n-1} r_{n}+\cdots .
$$

We call this dynamic programming "discounted DP". Furthermore, we can make the modified discounted DP's by assigning the translator $t(\cdot)$ the specified function such as $t(r)=(1-r) e^{r}$ or $t(r)=\log r$.

We have a following data from the Taxicab Problem due to Howard [6]. 
TABLE 4.1.1.

Data for general additive DP.

\begin{tabular}{|c|c|c|c|c|c|c|c|c|c|c|}
\hline \multirow{2}{*}{$\begin{array}{c}\text { state } \\
i\end{array}$} & \multirow{2}{*}{$\begin{array}{c}\text { action } \\
k\end{array}$} & \multicolumn{3}{|c|}{$\begin{array}{l}\text { transition } \\
\text { probability }\end{array}$} & \multicolumn{3}{|c|}{$\begin{array}{l}\text { stage-wise } \\
\text { reward }\end{array}$} & \multicolumn{3}{|c|}{$\begin{array}{l}\text { generalized } \\
\text { accumulator }\end{array}$} \\
\hline & & $p_{i 1}^{k}$ & $p_{i 2}^{k}$ & $p_{i 3}^{k}$ & $r_{i 1}^{k}$ & $r_{i 2}^{k}$ & $r_{i 3}^{k}$ & $\beta_{i 1}^{k}$ & $\beta_{i 2}^{k}$ & $\beta_{i 3}^{k}$ \\
\hline \multirow[t]{3}{*}{1} & 1 & $\frac{1}{2}$ & $\frac{1}{4}$ & $\frac{1}{4}$ & 10 & 4 & 8 & $\frac{95}{100}$ & $\frac{98}{100}$ & $\frac{98}{100}$ \\
\hline & 2 & $\frac{1}{16}$ & $\frac{3}{4}$ & $\frac{3}{16}$ & 8 & 2 & 4 & $\frac{90}{100}$ & $\frac{90}{100}$ & $\frac{93}{100}$ \\
\hline & 3 & $\frac{1}{4}$ & $\frac{1}{8}$ & $\frac{5}{8}$ & 4 & 6 & 4 & $\frac{98}{100}$ & $\frac{96}{100}$ & $\frac{98}{100}$ \\
\hline \multirow[t]{3}{*}{2} & 1 & $\begin{array}{l}1 \\
2\end{array}$ & 0 & $\frac{1}{2}$ & 14 & 0 & 18 & $\frac{85}{100}$ & $\frac{90}{100}$ & $\frac{95}{100}$ \\
\hline & 2 & $\frac{1}{16}$ & $\frac{7}{8}$ & $\frac{1}{16}$ & 6 & 16 & 8 & $\frac{80}{100}$ & $\frac{80}{100}$ & $\frac{95}{100}$ \\
\hline & 3 & $\frac{1}{3}$ & $\frac{1}{3}$ & $\frac{1}{3}$ & -5 & -5 & -5 & $\frac{95}{100}$ & $\frac{95}{100}$ & $\frac{95}{100}$ \\
\hline \multirow[t]{3}{*}{3} & 1 & $\frac{1}{4}$ & $\frac{1}{4}$ & $\frac{1}{2}$ & 10 & 2 & 8 & $\frac{75}{100}$ & $\frac{90}{100}$ & $\frac{95}{100}$ \\
\hline & 2 & $\frac{1}{8}$ & $\frac{3}{4}$ & $\frac{1}{8}$ & 6 & 4 & 2 & $\frac{95}{100}$ & $\frac{70}{100}$ & $\frac{80}{100}$ \\
\hline & 3 & $\frac{3}{4}$ & $\frac{1}{16}$ & $\frac{3}{16}$ & 4 & 0 & 8 & $\frac{95}{100}$ & $\frac{95}{100}$ & $\frac{95}{100}$ \\
\hline
\end{tabular}

Then the policy iteration algorithm (abbreviated, hereafter by PIA) described below gives an optimal stationary policy

$$
f=\left(\begin{array}{l}
1 \\
1 \\
3
\end{array}\right) .
$$

TABLE 4.1.2.

Solution by the PIA for general additive DP.

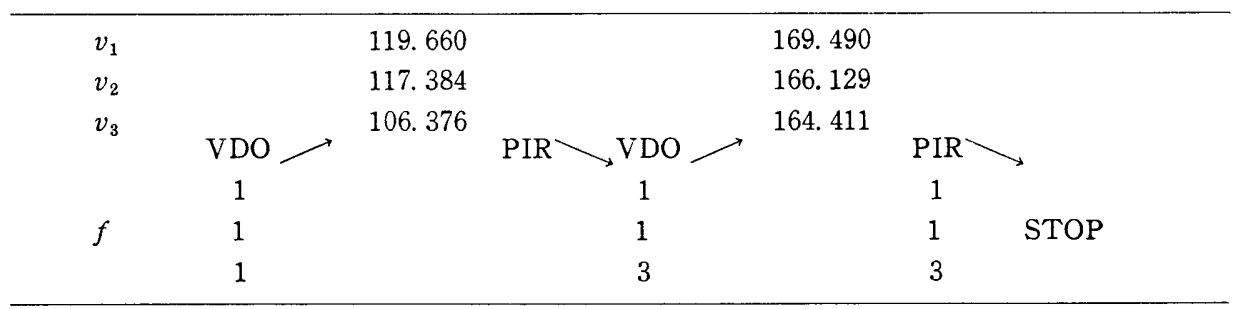

$\mathrm{VDO} \nearrow$ is the value determination operation.

PIR \ is the policy improvement routine.

However the PIA for discounted DP with same transition probability and same stage-wise reward as general additive DP yields an optimal stationary policy 


$$
g=\left(\begin{array}{l}
2 \\
2 \\
2
\end{array}\right) .
$$

TABLE 4.1.3.

Solution by the PIA for discounted DP with $\beta_{i j}^{k} \equiv 0.90$.

\begin{tabular}{|c|c|c|c|c|c|c|c|c|}
\hline$v_{1}$ & & 91.257 & & 119.439 & & 121.653 & & \\
\hline$v_{2}$ & & 97.551 & & 134.479 & & 135. 306 & & \\
\hline$v_{3}$ & & 89.967 & & 121. 927 & & 122.837 & & \\
\hline$g$ & 1 & & 2 & & 2 & & 2 & STOP \\
\hline & 1 & & 2 & & 2 & & 2 & \\
\hline
\end{tabular}

\section{EXAMPLE 2. (Multiplicative Additive System)}

We say that a dynamic programming $\{S, A, p, r\}$ has a multiplicative additive system if $\beta_{i j}^{k}=r_{i j}^{k}$ for any $(i, k, j) \in S A S$ and $t(r)=r$ in the recursive additive $\mathrm{DP}\{S, A, p, r, \beta, t\}$. We call this dynamic programming "multiplicative additive DP". Then, the reward system is given as follows:

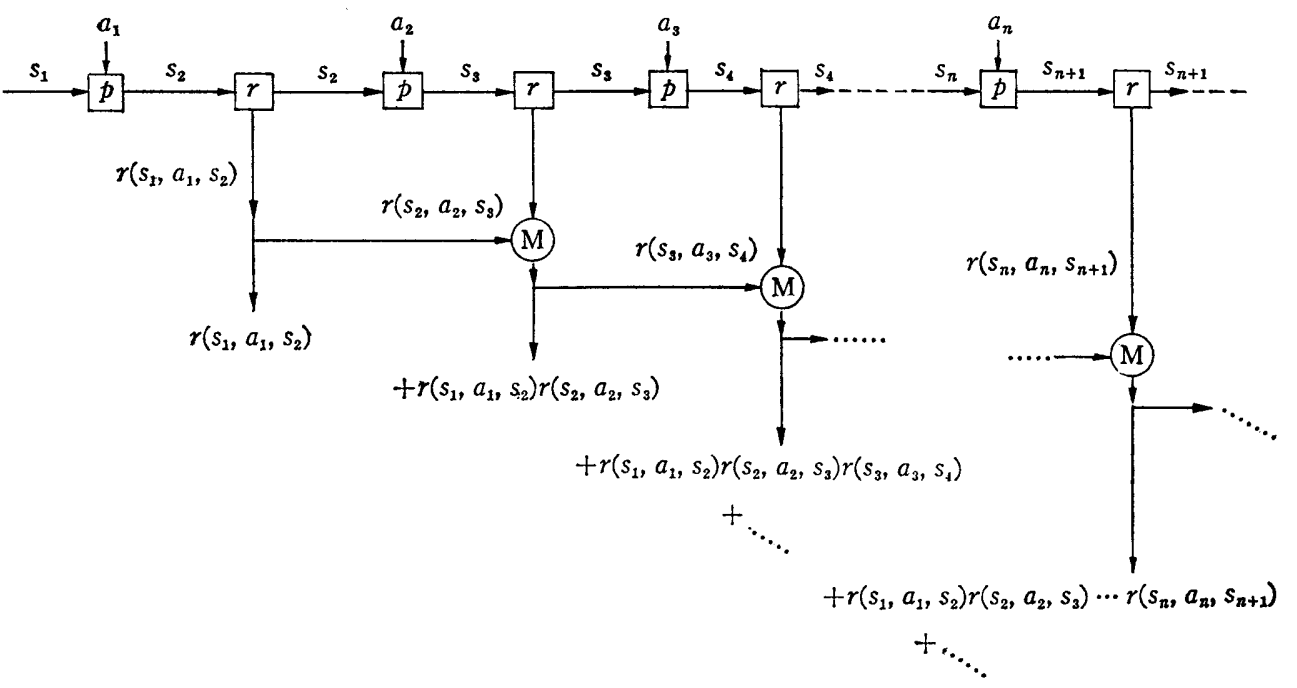

Fig. 2. Reward system of multiplicative additive DP.

This case leads us to maximization the expected value of the function

$$
V(h)=r_{1}+r_{1} r_{2}+r_{1} r_{2} r_{3}+\cdots+r_{1} r_{2} \cdots r_{n-1} r_{n}+\cdots,
$$

where $r_{k}$ and $h$ have the same meanings as Example 1. Then, Assumption (I) means $0 \leqq r_{i j}^{k}<1$ for any $(i, k, j) \in S A S$.

The stage-wise reward $r_{i j}^{k}$ in the following data satisfies Assumption (I). 
TABLE 4.2.1.

Data for multiplicative additive DP.

\begin{tabular}{|c|c|c|c|c|c|c|c|c|}
\hline \multirow{2}{*}{$\begin{array}{c}\text { state } \\
i\end{array}$} & \multirow{2}{*}{$\begin{array}{c}\text { action } \\
k\end{array}$} & \multicolumn{3}{|c|}{$\begin{array}{l}\text { transition } \\
\text { probability }\end{array}$} & \multicolumn{3}{|c|}{$\begin{array}{l}\text { stage-wise } \\
\text { reward }\end{array}$} & $\begin{array}{l}\text { generalized } \\
\text { accumulator }\end{array}$ \\
\hline & & $p_{i 1}^{k}$ & $p_{i 2}^{k}$ & $p_{i 3}^{k}$ & $r_{i 1}^{k}$ & $r_{i 2}^{k}$ & $r_{i 3}^{k}$ & $\beta_{i 1}^{k} \quad \beta_{i 2}^{k} \quad \beta_{i 3}^{k}$ \\
\hline \multirow{6}{*}{1} & \multirow{2}{*}{1} & 1 & 1 & 1 & 1 & 1 & 2 & \multirow{18}{*}{$\begin{array}{l}\beta_{i j}^{k}=r_{i j}^{k} \text { for } \\
\quad(i, k, j) \in S A S\end{array}$} \\
\hline & & 2 & 4 & 4 & 2 & 5 & 5 & \\
\hline & \multirow[b]{2}{*}{2} & 1 & 3 & 3 & 2 & 1 & 1 & \\
\hline & & 16 & 4 & 16 & 5 & 10 & 5 & \\
\hline & \multirow{2}{*}{3} & 1 & 1 & 5 & 1 & 3 & 1 & \\
\hline & & 4 & 8 & 8 & 5 & 10 & 5 & \\
\hline \multirow{6}{*}{2} & \multirow{2}{*}{1} & 1 & & 1 & 7 & 1 & 9 & \\
\hline & & 2 & 0 & 2 & 10 & 20 & 10 & \\
\hline & & 1 & 7 & 1 & 2 & 4 & 2 & \\
\hline & 2 & 16 & 8 & 16 & 5 & 5 & 5 & \\
\hline & & 1 & 1 & 1 & 1 & 1 & 1 & \\
\hline & 3 & 3 & 3 & 3 & 20 & 20 & $\overline{20}$ & \\
\hline \multirow{6}{*}{3} & & 1 & 1 & 1 & 1 & 1 & 2 & \\
\hline & 1 & 4 & 4 & 2 & 2 & 10 & 5 & \\
\hline & & 1 & 3 & 1 & 3 & 1 & 1 & \\
\hline & 2 & 8 & 4 & 8 & $\overline{10}$ & 5 & $\overline{10}$ & \\
\hline & & 3 & 1 & 3 & 1 & 1 & 2 & \\
\hline & 3 & 4 & 16 & 16 & 5 & 20 & 5 & \\
\hline
\end{tabular}

The calculations by PIA are shown in the following:

TABLE 4.2 .2

Solution by the PIA for multiplicative additive DP.

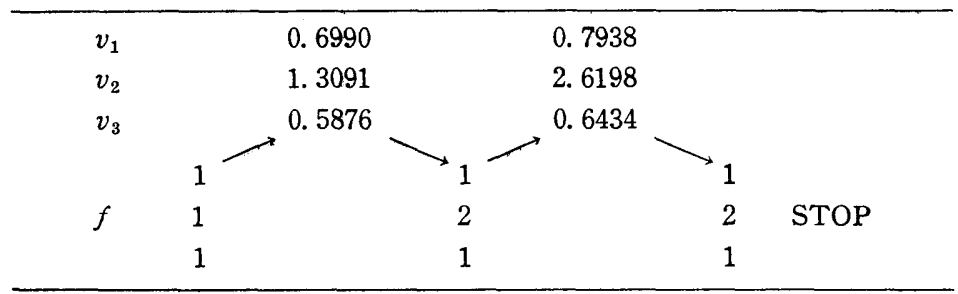

Then we get an optimal stationary policy

$$
f=\left(\begin{array}{l}
1 \\
2 \\
1
\end{array}\right)
$$

for this multiplicative additive DP. But the optimal one for discounted DP with $\beta=0.95$ is

$$
g=\left(\begin{array}{l}
2 \\
2 \\
2
\end{array}\right)
$$


TABLE 4.2.3.

Solution by the PIA for discounted DP with $\beta_{i j}^{k} \equiv 0.95$.

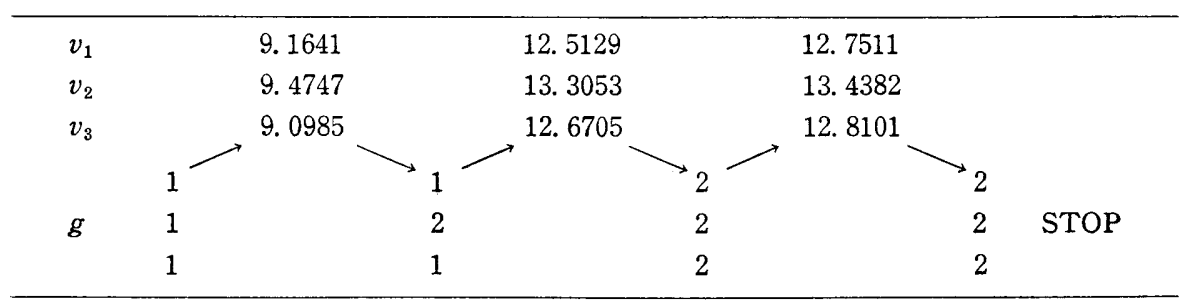

EXAMPle 3. (Divided Additive System)

We say that a dynamic programming $\{S, A, p, r\}$ has a divided additive system if $\beta_{i j}^{k}=1 / r_{i j}^{k}$ for any $(i, k, j) \in S A S$ and $t(r)=r$ in the recursive additive $\operatorname{DP}\{S, A, p$, $r, \beta, t\}$. We call this dynamic programming "divided additive DP". Then, we have the following reward system:

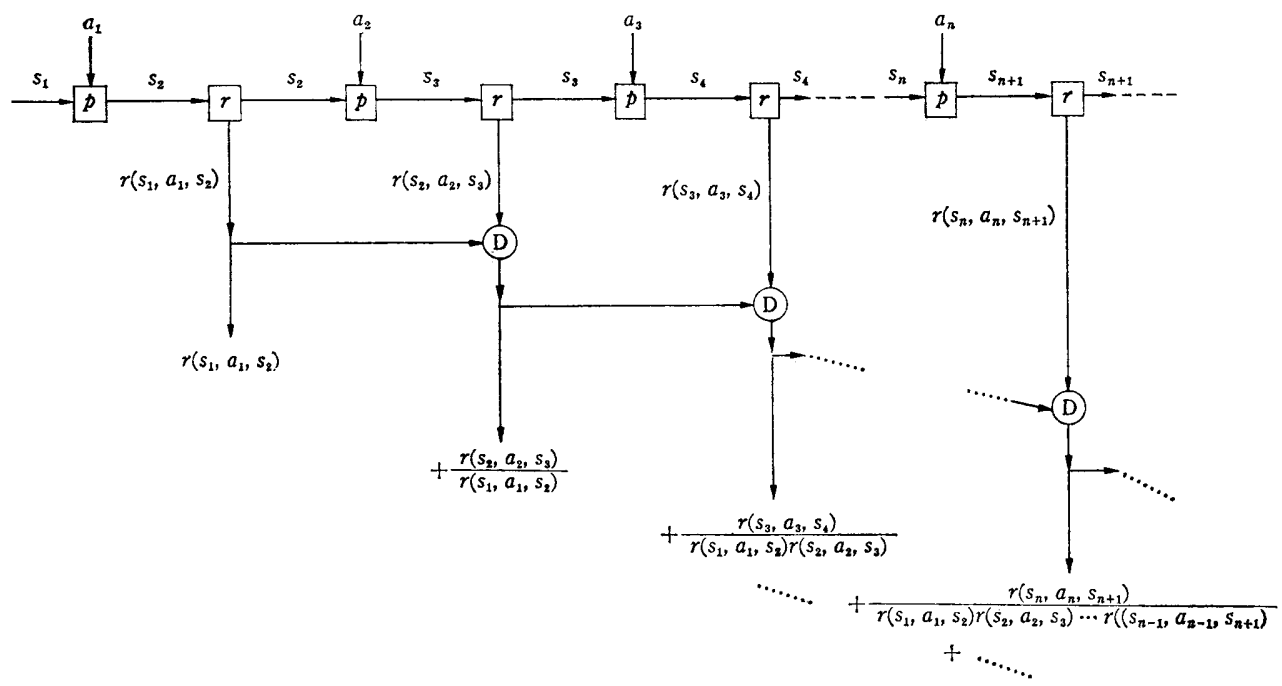

Fig. 3. Reward system of divided additive DP.

Here (D) means $\frac{v}{u} \longrightarrow \stackrel{\downarrow}{\downarrow} \underset{\frac{w}{u v}}{\downarrow}$

We have an optimization problem whose objective function is the expected return of the function

$$
V(h)=r_{1}+\frac{r_{2}}{r_{1}}+\frac{r_{3}}{r_{1} r_{2}}+\cdots+\frac{r_{n}}{r_{1} r_{2} \cdots r_{n-1}}+\cdots,
$$

where $h=\left(s_{1}, a_{1}, s_{2}, a_{2}, \cdots\right), r_{k}=r_{s_{k^{3} k+1}}^{a_{k}}$ for $k \geqq 1$. 
A dynamic programming problem $\{S, A, p, r, t\}$ is called "modified divided additive DP" if $\beta_{i j}^{k}=1 / r_{i j}^{k}$ for $(i, k, j) \in S A S$ in recursive additive $\operatorname{DP}\{S, A, p, r, \beta, t\}$. Then in the modified divided additive DP

$$
V(h)=r_{1}+\frac{t\left(r_{2}\right)}{r_{1}}+\frac{t\left(r_{3}\right)}{r_{1} r_{2}}+\cdots+\frac{t\left(r_{n}\right)}{r_{1} r_{2} \cdots r_{n-1}}+\cdots
$$

We can see, in Bellman's book ([1], Chap. I), an example of modified additive DP, which is restricted to the finite horizon and deterministic case.

If $r_{i j}^{k}>1$ for any $(i, k, j) \in S A S$, then Assumption (I) is statisfied. The stagewise rewards in the following satisfy Assumption(I).

TABLE 4.3.1.

Data for divided additive DP.

\begin{tabular}{|c|c|c|c|c|c|c|c|c|}
\hline \multirow{2}{*}{$\begin{array}{l}\text { state } \\
\quad i\end{array}$} & \multirow{2}{*}{$\begin{array}{c}\text { action } \\
k\end{array}$} & \multicolumn{3}{|c|}{$\begin{array}{l}\text { transition } \\
\text { probability }\end{array}$} & \multicolumn{3}{|c|}{$\begin{array}{l}\text { stage-wise } \\
\text { reward }\end{array}$} & $\begin{array}{c}\text { generalized } \\
\text { accumulator }\end{array}$ \\
\hline & & $p_{i 1}^{k}$ & $p_{i 2}^{k}$ & $p_{i 3}^{k}$ & $r_{i 1}^{k}$ & $r_{i 2}^{k}$ & $r_{i 3}^{k}$ & $\beta_{i 1}^{k} \quad \beta_{i 2}^{k} \quad \beta_{i 3}^{k}$ \\
\hline \multirow{6}{*}{1} & \multirow{2}{*}{1} & 1 & 1 & 1 & 3 & 6 & 7 & \multirow{18}{*}{$\begin{aligned} \beta_{i j}^{k}= & \frac{1}{r_{i j}^{k}} \\
& \text { for }(i, j, k)\end{aligned}$} \\
\hline & & $\overline{2}$ & 4 & 4 & 2 & 5 & 5 & \\
\hline & \multirow{2}{*}{2} & 1 & 3 & 3 & 7 & 11 & 6 & \\
\hline & & 16 & 4 & 16 & 5 & 10 & 5 & \\
\hline & \multirow{2}{*}{3} & 1 & 1 & 5 & 6 & 13 & 6 & \\
\hline & & 4 & 8 & 8 & 5 & 10 & 5 & \\
\hline \multirow{6}{*}{2} & 1 & 1 & 0 & 1 & 17 & 21 & 19 & \\
\hline & 1 & 2 & 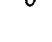 & 2 & 10 & 20 & 10 & \\
\hline & $?$ & 1 & 7 & 1 & 7 & 9 & 26 & \\
\hline & 2 & 16 & 8 & 16 & 5 & 5 & 25 & \\
\hline & 3 & 1 & 1 & 1 & 21 & 21 & 21 & \\
\hline & 3 & 3 & 3 & 3 & 20 & 20 & 20 & \\
\hline \multirow{6}{*}{3} & & 1 & 1 & 1 & 3 & 11 & 7 & \\
\hline & 1 & 4 & 4 & 2 & 2 & 10 & 5 & \\
\hline & & 1 & 3 & 1 & 13 & 6 & 11 & \\
\hline & 2 & 8 & 4 & 8 & 10 & 5 & 10 & \\
\hline & & 3 & 1 & 3 & 6 & 21 & 7 & \\
\hline & 3 & 4 & 16 & 16 & 5 & 20 & 5 & \\
\hline
\end{tabular}

The calculation by PIA for this divided additive DP is given as follows:

TABLE 4.3.2.

Solution by the PIA for divided additive DP.

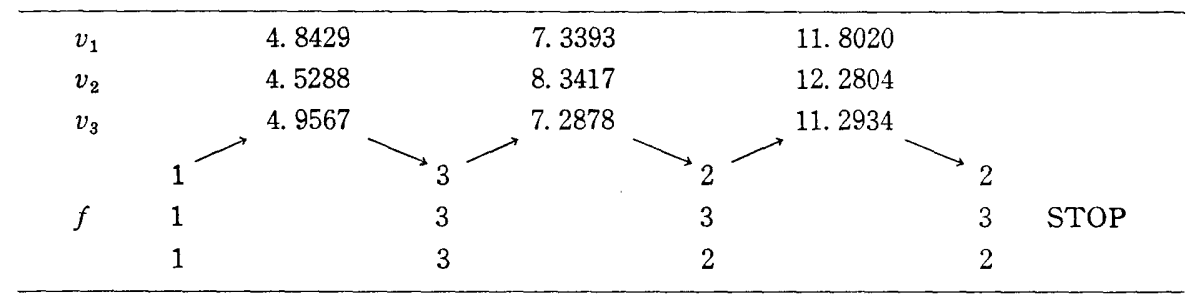


We have an optimal stationary policy

$$
f=\left(\begin{array}{l}
2 \\
3 \\
2
\end{array}\right) .
$$

On the other hand, if transition probability $p_{i j}^{k}$ and state-wise reward $r_{i j}^{k}$ are the same as ones for this divided additive DP respectively, the optimal stationary policy for discounted DP with discount factor $\beta=0.95$ is

$$
g=\left(\begin{array}{l}
2 \\
2 \\
2
\end{array}\right) .
$$

Following table shows this calculation:

TABLE 4.3.3.

Solution by the PIA for discounted DP with $\beta_{i j}^{k} \equiv 0.95$.

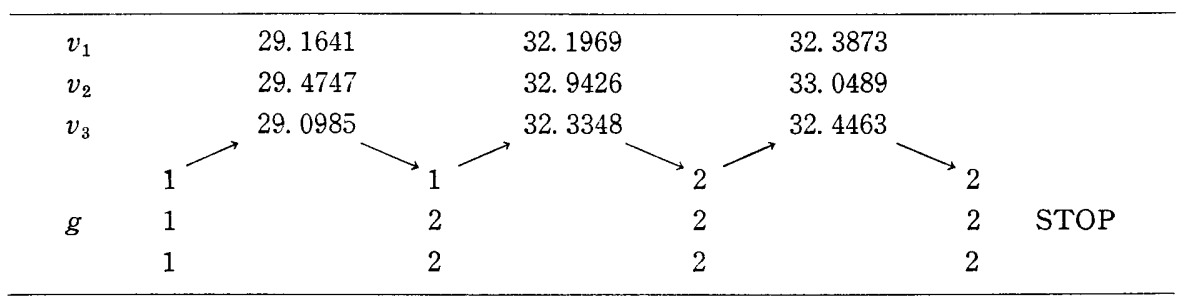

\section{ExAmple 4. (Exponential Additive System)}

We say that a dynamic programming $\{S, A, p, r\}$ has an exponential additive system if $\beta_{i j}^{k}=e^{r_{i j}^{k}}$ for any $(i, k, j) \in S A S$ and $t(r)=r$ in the recursive additive DP $\{S, A, p$, $r, \beta, t\}$. We call this dynamic programming "exponential additive DP". The reward system of the exponential additive DP is just describled by Fig. 3 except for

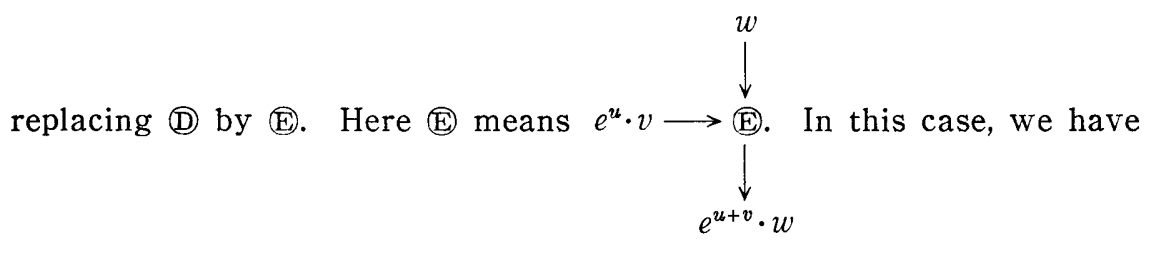

$$
V(h)=r_{1}+e^{r_{1}} \cdot r_{2}+e^{r_{1}+r_{2}} \cdot r_{3}+\cdots+e^{r_{1}+r_{2} \cdots r_{n-1}} \cdot r_{n}+\cdots,
$$

where $h=\left(s_{1}, a_{1}, s_{2}, a_{2}, \cdots\right), r_{k}=r_{s_{k} s_{k+1}}^{a_{k}}$ for $k \geqq 1$. Moreover, if $t(r)=(1-r) e^{r}, \beta_{i j}^{k}=e^{r_{i j}^{k}}$ for any $(i, k, j) \in S A S$ in recursive additive DP, we have another objective function

$$
\begin{array}{r}
V^{\prime}(h)=\left(1-r_{1}\right) e^{r_{1}}+\left(1-r_{2}\right) e^{r_{1}+r_{2}}+\left(1-r_{3}\right) e^{r_{1}+r_{2}+r_{3}} \\
+\cdots+\left(1-r_{n}\right) e^{r_{1}+r_{2}+\cdots+r_{n}}+\cdots .
\end{array}
$$

We call this dynamic programming $\{S, A, p, r\}^{\prime}$ " modified exponential additive DP". In these exponential additive DP's, Assumption (I) means $r_{i j}^{k}<0$ for any $(i, k, j) \in S A S$. 
We can find an example of modified exponential additive DP in Bellman's book ([1], Chap. III). The stage-wide rewards illustrated in the following table satisfy Assumption (I).

TABLE 4.4.1.

Data for exponential additive DP.

\begin{tabular}{|c|c|c|c|c|c|c|c|c|}
\hline \multirow{2}{*}{$\begin{array}{c}\text { state } \\
i\end{array}$} & \multirow{2}{*}{$\begin{array}{c}\text { action } \\
k\end{array}$} & \multicolumn{3}{|c|}{$\begin{array}{l}\text { transition } \\
\text { probability }\end{array}$} & \multicolumn{3}{|c|}{$\begin{array}{l}\text { stage-wise } \\
\text { reward }\end{array}$} & $\begin{array}{l}\text { generalized } \\
\text { accumulator }\end{array}$ \\
\hline & & $p_{21}^{k}$ & $p_{i 2}^{k}$ & $p_{i 3}^{k}$ & $r_{i 1}^{k}$ & $r_{i 2}^{k}$ & $r_{i 3}^{k}$ & $\beta_{i 1}^{k} \quad \beta_{i 2}^{k} \quad \beta_{i 3}^{k}$ \\
\hline \multirow{6}{*}{1} & \multirow{2}{*}{1} & 1 & 1 & 1 & 1 & 1 & 2 & \multirow{18}{*}{$\begin{array}{l}\beta_{i j}^{k}=e^{r_{i j}^{k}} \\
\quad \text { for }(i, k, j)\end{array}$} \\
\hline & & 2 & 4 & 4 & 2 & 5 & 5 & \\
\hline & \multirow[b]{2}{*}{2} & 1 & 3 & 3 & 2 & 1 & 1 & \\
\hline & & 16 & 4 & 16 & 5 & 10 & 5 & \\
\hline & \multirow[b]{2}{*}{3} & 1 & 1 & 5 & 1 & 3 & 1 & \\
\hline & & 4 & 8 & 8 & 5 & 10 & 5 & \\
\hline \multirow{6}{*}{2} & 1 & 1 & 0 & 1 & 7 & 1 & 9 & \\
\hline & & 2 & 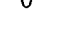 & 2 & $-\frac{10}{10}$ & 20 & $-\frac{10}{10}$ & \\
\hline & 2 & 1 & 7 & 1 & 2 & 4 & 2 & \\
\hline & 2 & 16 & 8 & 16 & 5 & 5 & 5 & \\
\hline & & 1 & 1 & 1 & 1 & 1 & 1 & \\
\hline & 3 & 3 & $\overline{3}$ & 3 & 20 & 20 & 20 & \\
\hline \multirow{6}{*}{3} & \multirow{2}{*}{1} & 1 & 1 & 2 & 1 & 1 & 2 & \\
\hline & & 4 & 4 & 4 & 2 & 10 & 5 & \\
\hline & \multirow{2}{*}{2} & 1 & 3 & 1 & 3 & 1 & 1 & \\
\hline & & 8 & 4 & 8 & 10 & 5 & 10 & \\
\hline & \multirow{2}{*}{3} & 3 & 1 & 3 & 1 & 1 & 2 & \\
\hline & & 4 & 16 & 16 & 5 & 20 & 5 & \\
\hline
\end{tabular}

The optimal policy for the exponential additive DP which is stationary is given as follows:

TABLE 4.4.2.

Solution by the PIA for exponential additive DP.

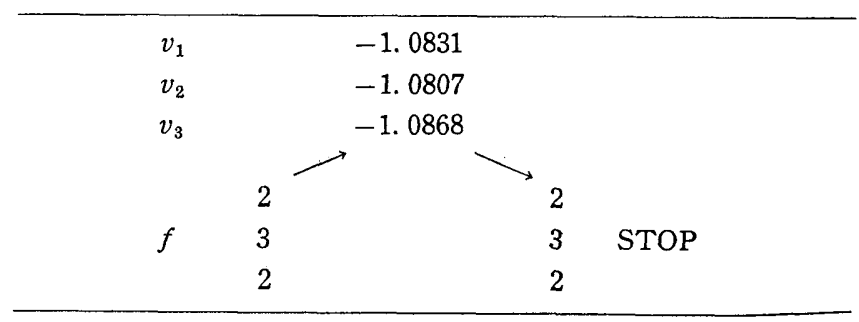

Moreover, we obtain following tables: 
TABLE 4.4.3.

Solution by the PIA for discounted DP with $\beta_{i j}^{k} \equiv 0.95$.

\begin{tabular}{lllll}
$v_{1}$ & & & \\
$v_{2}$ & & & \\
$v_{3}$ & & & \\
& 2.2 .0935 & & \\
-2.2093 & & 3 & STOP \\
& 3 & & 2 & \\
\hline
\end{tabular}

TABLE 4.4.4.

Solution by the PIA for modified exponential additive DP with $t(r)=(1-r) e^{r}$

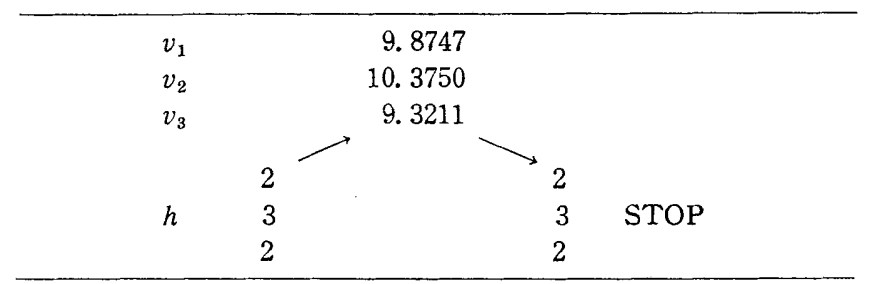

Of course the transition probability $p_{i j}^{k}$ and stage-wise reward $r_{i j}^{k}$ are common in these three DP's.

Note that each optimal policy is common one;

$$
f=g=h=\left(\begin{array}{l}
2 \\
3 \\
2
\end{array}\right]
$$

and that policy iteration algorithm converges at first cycle respectively.

EXAMPLE 5. (Logarithmic Additive System)

We say that a dynamic programming $\{S, A, p, r\}$ has a logarithmic additive system if $\beta_{i j}^{k}=\log r_{i j}^{k}$ for any $(i, k, j) \in S A S$ and $t(r)=r$ in the recursive additive DP $\{S, A$, $p, r, \beta, t\}$. We call this dynamic programming "logarithmic additive DP". The reward system of logarithmic additive DP is just described by Fig. 3 except for replacing (D) by (1). Here, (1) means $(\log u) v \rightarrow \stackrel{(1)}{ }_{(\log u)(\log v) w}^{w}$ Then, we have
$V(h)=r_{1}+\left(\log r_{1}\right) r_{2}+\left(\log r_{1}\right)\left(\log r_{2}\right) r_{3}$
$+\cdots+\left(\log r_{1}\right)\left(\log r_{2}\right) \cdots\left(\log r_{n-1}\right) r_{n}+\cdots$,

where $h=\left(s_{1}, a_{1}, s_{2}, a_{2}, \cdots\right), r_{k}=r_{s^{s} k+1}^{a_{k}}$ for $k \geqq 1$. Moreover, if $t(r)=\log r, \beta_{i j}^{k}=\log r_{i j}^{k}$ for any $(i, k, j) \in S A S$ in recursive additive DP, we have another logarithmic additive 
DP where objective function is the expected return of the function

$$
\begin{array}{r}
V^{\prime}(h)=\log r_{1}+\left(\log r_{1}\right)\left(\log r_{2}\right)+\left(\log r_{1}\right)\left(\log r_{2}\right)\left(\log r_{3}\right) \\
+\cdots+\left(\log r_{1}\right)\left(\log r_{2}\right) \cdots\left(\log r_{n}\right)+\cdots .
\end{array}
$$

We call this dynamic programming "modified logarithmic additive DP". If $1 \leqq r_{i j}^{k}<e$ for any $(i, k, j) \in S A S$, then Assumption (I) is satisfied for these logarithmic additive DP's. The following data satisfies Assumption (I).

TABLE 4.5.1.

\begin{tabular}{|c|c|c|c|c|c|c|c|c|}
\hline \multirow{2}{*}{$\begin{array}{c}\text { state } \\
\qquad i\end{array}$} & \multirow{2}{*}{$\begin{array}{c}\text { action } \\
k\end{array}$} & \multicolumn{3}{|c|}{$\begin{array}{l}\text { transition } \\
\text { probability }\end{array}$} & \multicolumn{3}{|c|}{$\begin{array}{l}\text { stage-wise } \\
\text { reward }\end{array}$} & $\begin{array}{c}\text { generalized } \\
\text { accumulator }\end{array}$ \\
\hline & & $p_{i 1}^{k}$ & $p_{i 2}^{k}$ & $p_{i 3}^{k}$ & $r_{i 1}^{k}$ & $r_{i 2}^{k}$ & $r_{i 3}^{k}$ & $\beta_{i 1}^{k} \quad \beta_{i 2}^{k} \quad \beta_{i 3}^{k}$ \\
\hline \multirow[t]{3}{*}{1} & 1 & $\frac{1}{2}$ & $\frac{1}{4}$ & $\frac{1}{4}$ & 2.3 & 2. 7 & 2.4 & \multirow{9}{*}{$\begin{array}{l}\beta_{i j}^{k}=\log r_{i j}^{k} \\
\quad \text { for }(i, k, j)\end{array}$} \\
\hline & 2 & $\frac{1}{16}$ & $\frac{3}{4}$ & $\frac{3}{16}$ & 2.7 & 2. 3 & 2.6 & \\
\hline & 3 & $\frac{1}{4}$ & $\frac{1}{8}$ & $\begin{array}{l}5 \\
8\end{array}$ & 2.5 & 2.4 & 2. 6 & \\
\hline \multirow[t]{3}{*}{2} & 1 & $\frac{1}{2}$ & 0 & $\frac{1}{2}$ & 2.7 & 2. 3 & 2. 4 & \\
\hline & 2 & $\frac{1}{16}$ & $\frac{7}{8}$ & $\frac{1}{16}$ & 2. 6 & 2.4 & 2. 7 & \\
\hline & 3 & $\frac{1}{3}$ & $\frac{1}{3}$ & $\frac{1}{3}$ & 2.4 & 2.1 & 2.5 & \\
\hline \multirow[t]{3}{*}{3} & 1 & $\frac{1}{4}$ & $\frac{1}{4}$ & $\frac{1}{2}$ & 2.6 & 2.5 & 2.7 & \\
\hline & 2 & $\frac{1}{8}$ & $\frac{3}{4}$ & $\frac{1}{8}$ & 2.7 & 2.6 & 2. 4 & \\
\hline & 3 & $\frac{3}{4}$ & $\frac{1}{16}$ & $\frac{3}{16}$ & 2.6 & 2.7 & 2.5 & \\
\hline
\end{tabular}

Data for logarithmic additive DP.

The PIA yields optimal policies associated with logarithmic additive DP, discounted DP with $\beta_{i j}^{k} \equiv \beta=0.95$ and discounted DP with $t(r)=\log r, \beta_{i j}^{k} \equiv 0.95$ respectively.

TABLE 4.5.2.

\begin{tabular}{|c|c|c|c|c|}
\hline$v_{1}$ & & 19. 2080 & & \\
\hline$v_{2}$ & & 19.1090 & & \\
\hline$v_{3}$ & & 19. 7274 & & \\
\hline & 3 & & 3 & \\
\hline$f$ & 1 & & 1 & STOP \\
\hline & 1 & & 1 & \\
\hline
\end{tabular}

Solution by the PIA for logarithmic additive DP. 
TABLE 4.5.3.

Solution by the PIA for discounted DP with $\beta_{i j}^{k} \equiv 0.95$.

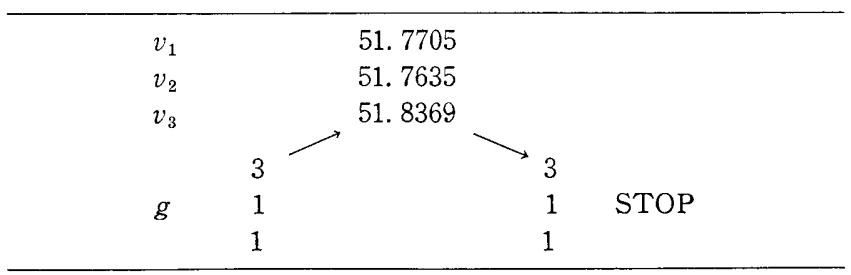

TABLE 4.5.4.

Solution by the PIA for modified discounted DP with $\beta_{i j}^{k}=0.95, t(r)=\log r$

\begin{tabular}{llr}
$v_{1}$ & & 19.0064 \\
$v_{2}$ & & \\
$v_{3}$ & \\
& 3 & \\
19.0025 & \\
19.0318 & \\
3 & & 1 \\
1 & & 1 \\
\hline
\end{tabular}

In these three DPs we have a common optimal stationary policy

$$
f=g=h=\left(\begin{array}{l}
3 \\
1 \\
1
\end{array}\right) .
$$

Throughout above five examples, we have a remark that the ranges of the value $r_{i j}^{k}$ for $(i, k, j) \in S A S$ are $(-\infty, 0),[0,1),(1, \infty),[1, e)$ corresponding to exponential additive, multiplicative additive, divided additive and logarithmic additive DPs respectively. By virtue of these examples, it is natural that we call $\beta=\left(\beta_{i j}^{k}\right)$ a generalized accumulator.

\section{Additional comments.}

In this section we will show that the recursive additive DP satisfying Assumption (I) can be reduced to a DP with an absorved state. We will give some comments on recursive additive DP.

Let $\{S, A, p, r, \beta, t\}$ be a recursive additive DP satisfying Assumption (I). Let $0 \notin S$ be a fictitious state and $K_{0}=\{1\}$. That is, the set of available actions at state 0 consists of a single action 1 . We define $\bar{S}, \bar{A}, \bar{p}$ and $\bar{r}$ as follows :

$$
\begin{aligned}
& \bar{S}=S \cup\{0\}, \\
& \bar{A}=\{K_{0}, \underbrace{A, A, \cdots, A}_{N \text { factors }}\}, \\
& \bar{p}_{i j}^{k}= \begin{cases}\bar{p}_{00}^{1}=1, \\
\bar{p}_{i 0}^{k}=1-\sum_{j \in S} \beta_{i j}^{k} p_{i j}^{k} & \text { for }(i, k) \in S \times A, \\
\bar{p}_{i j}^{k}=\beta_{i j}^{k} p_{i j}^{k} & \text { for } \quad(i, k, j) \in S \times A \times S,\end{cases}
\end{aligned}
$$




$$
r_{i}^{k}= \begin{cases}0 & \text { for } \quad(i, k)=(0,1), \\ \sum_{j=S} p_{i j}^{k} t\left(r_{i j}^{k}\right) & \text { for } \quad(i, k) \in S \times A .\end{cases}
$$

Then in a new-defined $\operatorname{DP}\{\bar{S}, \bar{A}, \bar{p}, \bar{r}\}$ the stage-wise reward $\bar{r}=\left(\bar{r}_{i}^{k}\right)$ does not depend on next state $j \in S$ but the set of available actions depends on current state $i \in S$.

LEMMA 4.1. Let $\{S, A, p, r, \beta, t\}$ be a recursive additive $D P$ satisfying Assumption (I). Then, $\{\bar{S}, \bar{A}, \bar{p}, \bar{r}\}$ is a DP with an absorved state 0 . Furthermore any policy $\pi$ in recursive additive $D P\{S, A, p, r, \beta, t\}$ can be extended to the policy $\bar{\pi}$ in reduced $D P\{\bar{S}, \bar{A}, \bar{p}, \bar{r}\}$ such that

$$
V(\pi)(i)=\bar{V}(\bar{\pi})(i) \quad \text { on } \quad S, \quad \bar{V}(\bar{\pi})(0)=0 .
$$

Conversely, any policy $\bar{\pi}$ in reduced $D P\{\bar{S}, \bar{A}, \bar{p}, \bar{r}\}$ can be regarded as a policy $\pi$ in recursive additive $D P\{S, A, p, r, \beta, t\}$ such that

$$
\bar{V}(\bar{\pi})(i)=V(\pi)(i) \text { on } S .
$$

In this case $\bar{V}(\bar{\pi})(0)=0$ is trivial consequence.

Proof. Let $P$ be $N \times N$ stochastic matrix and $\bar{P}=\left[\begin{array}{ll}1 & 0 \\ x & P\end{array}\right]$ be $(N+1) \times(N+1)$ matrix. Then we have $\bar{P}^{n}=\left[\begin{array}{cc}1 & 0 \\ x_{n} & P^{n}\end{array}\right]$ for each $n \geqq 1$. The proof is an immediate consequence of this result. The details are ommitted.

It should be noted that the generalized accumulator $\beta=\left(\beta_{i j}^{k}\right)$ in recursive additive DP is embedded into the transition law $\bar{p}=\left(\bar{p}_{i j}^{k}\right)$ of reduced DP. Hence it is rather difficult to get five examples in Section 4 from the reduced one.

There is a kind of sensitivity analysis as follows: Is there any relation between general additive $\operatorname{DP}\{S, A, p, r, \beta\}$ and recursive additive $\operatorname{DP}\{S, A, p, r, \beta, t\}$ ? In other words, what is a sufficient condition on $t=t(\cdot)$ for optimal policy in the former to remain optimal in the latter? The analysis of this sensitivity will require a detail analysis of Howard's policy iteration algorithm.

Acknowledgement. The author wishes to express his hearty thanks to Professor T. Kitagawa and Doctor N. Furukawa for some useful comments.

\section{References}

[1] R. Bellman, Dynamic Programming. Princeton Univ. Press. (1957).

[2] D. Blackwell, Discrete Dynamic Programming. Ann. Math. Stat., 33, (1962), 719-726.

[3] D. Blackwell, Discounted Dynamic Programming. Ann. Math. Stat., 36, (1965), 226235.

[4] D. Blackwell, Positive Dynamic Programming. Proc. Fifth Berkeley Symp. Math. Statist. Probability, Vol. I, Univ. of California, Press. Berkeley, California, (1967), $415-418$.

[5] N. Furukawa and S. Iwamoto, Markovian Decision Processes with Recursive Reward Functions. Bull. Math. Statist., 15, No. 3-4 (1973), 79-91.

[6] R. A. Howard, Dynamic Programming and Markov Processes. M.I.T. Press, Cambridge, Massachusetts, (1960).

[7] R. E. Strauch, Negative Dynamic Programming. Ann. Math. Stat., 37 (1966), 871-890. 